\title{
Area-Preserving Parameterizations for Spherical Ellipses
}

\author{
Ibón Guillén ${ }^{1,2}$, Carlos Ureña ${ }^{3}$, Alan King $^{4}$, Marcos Fajardo ${ }^{4}$, Iliyan Georgiev ${ }^{4}$, \\ Jorge López-Moreno ${ }^{2}$, Adrian Jarabo ${ }^{1}$ \\ ${ }^{1}$ Graphics \& Imaging Lab \\ Instituto de Investigación en Ingeniería de Aragón (I3A) \\ Universidad de Zaragoza, Mariano Esquillor s/n, 50018, Zaragoza, Spain. \\ Tel.+34-976762353, e-mail: ibon@unizar.es \\ ${ }^{2}$ Universidad Rey Juan Carlos \\ ${ }^{3}$ Universidad de Granada \\ ${ }^{4}$ Solid Angle
}

\section{Abstract}

We present new methods for efficient estimation of the radiance incoming from disk-shaped light sources. For that, we introduce three novel areapreserving mappings for the projected solid angle of the disk. Our methods significantly improve over previous work, and have been implemented in a mainstream production-oriented render engine.

\section{Introduction}

Generating realistic synthetic images from purely virtual scene descriptions, in the process known as rendering, requires an accurate simulation light transport, including its emission, scattering and capture. Currently, due to the complexity and high dimensionality of the problem, the preferred method employed to carry these computations is Monte Carlo integration [5]. This method can be understood as a brute-force stochastic simulation of the underlying light processes, in order to obtain a close enough estimation of the complete light transport. For more in-detailed explanations about light transport estimation techniques and its applications we refer the reader to [8].

One of the most important effects required for achieving realistic rendering is the use of emissive surfaces (usually called area lights).In order to compute the radiance incoming at a surface via Monte Carlo integration, we need to sample to projected surface of the area light as uniformly as possible. This is a non-trivial problem for light sources of arbitrary shapes [2].

The most straightforward rule for distributing the samples lighting, uniformly in the light source surface, results in (potentially) infinite variance in due to the cancellation of the geometric attenuation terms. A much better alternative is to distribute the samples over the spherical projection of the light source surface, its solid angle.

In this work we develop a set of good sampling methods for the case of disk-shaped light sources. For this kind of emitters, the best pre-existing technique bases on using rejection sampling over a bounding quad [6] (i.e. discarding the samples generated outside the actual disk). While considerably better than direct surface-area sampling, rejection sampling techniques make it difficult to achieve good sample stratification across higher dimensions, important to guarantee good convergence in Monte Carlo. In contrast, our method allow for a explicit stratification of the disk's solid angle projection. Our work has been published in Computer Graphics Forum [7], and presented at the Eurographics Symposium on Rendering 2017.

\section{Our method}

One of our key observations is that the projection of an arbitrarily oriented disk into a sphere results in a spherical ellipse [3]. The other key insight of our method is to use of the so called Hat-Box theorem [1], by which the area of a region on the sphere between two parallels is equal to its projection onto a perpendicularly aligned unit cylinder. With these two observations we simplify the problem from sampling the spherical ellipse surface into sampling its planar projection on the bounding cylinder.

We develop three roughly equivalent solid angle mappings, which we denominate the parallel, polar and low-distortion. The result of employing the different mapping is illustrated in Figure 1. The lowdistortion mapping has the best properties for its use in Monte Carlo, since it is both efficient to evaluate and preserves the discrepancy properties of the sampling sequence. The complete mathematical details can be consulted on the main document [7]. 


\section{Implementation and results}

While our formulation provides a closed-form solution for the calculation of the complete solid angle, its inversion must be performed either numerically or by using a precalculated table of partial solid angle values. The second allows for very efficient sampling, introducing minimal error. In-depth implementation details can be found in [7]. Our low-distorsion mapping, and its associated sampling procedures, are currently implemented in the Arnold renderer [4]; a commercial software for movie production that recently received the Technical Award of the Academy (i.e. an Oscar). In Figure 2 we provide a comparison between the previous surface-area sampling and our improved solid angle sampling.

\section{Conclusions}

We have presented three new area-preserving mappings that allow uniform solid angle sampling of oriented disk-shaped light sources. Our mappings outperform existing solid angle sampling techniques for disks, without imposing restrictions on the sampling sequence or with significant overhead, and has been integrated in the Arnold renderer. While this work only considers circular disks, our approach could be extrapolated to other shapes whose subtended solid angle is also a spherical ellipse, such as elliptical disks and ellipsoids.

\section{REFERENCES}

[1]. ARCHIMEDES: On the Sphere and Cylinder. -225.

[2]. ARVO, J.: Stratified sampling of 2-manifolds. SIGGRAPH 2001 Course Notes, 2001, 29(2).

[3]. BOOTH, J.: On the rectification and quadrature of the spherical ellipse. The London, Edinburgh, and Dublin Philosophical Magazine and Journal of Science, 1844, 25(163), 18-38.
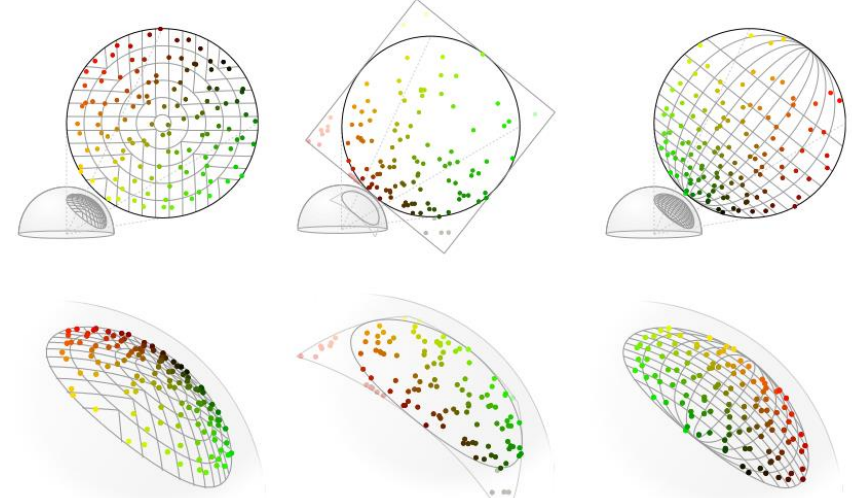
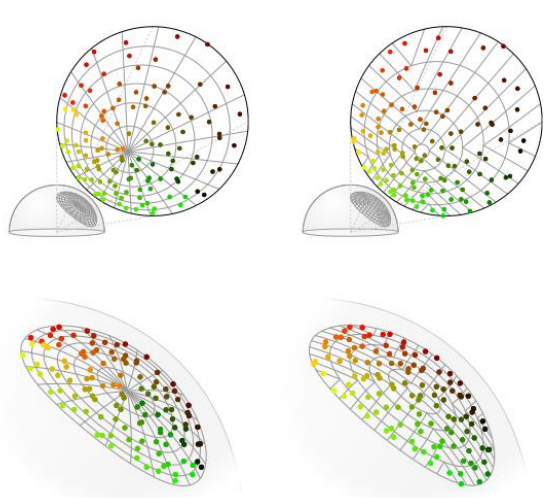

[4]. FAJARDO, M.: Global Illumination Across Industries: Ray tracing solution for film production rendering. ACM SIGGRAPH 2010 Talks, 2010.

[5]. FASCIONE, L., HANIKA, J., FAJARDO, M., CHRISTENSEN, P., BURLEY, B., GREEN, B.: Path tracing in production-part 1: production renderers. ACM SIGGRAPH 2017 Courses, 2017.

[6]. GAMITO M. N.: Solid angle sampling of disk and cylinder lights. Computer Graphics Forum, 2016, 34(4), 25-36.

[7]. GUILLÉN I., UREÑA C., KING A., FAJARDO M., GEORGIEV I., LÓPEZ-MORENO J., JARABO A.: Area-preserving parameterizations for spherical ellipses. Computer Graphics Forum, 2017, 36(4), 179-187.

[8]. PHARR, M., JAKOB, W., HUMPHREYS, G.: Physically Based Rendering: From Theory To Implementation, 3rd ed. USA: Morgan Kaufmann Inc., 2016.

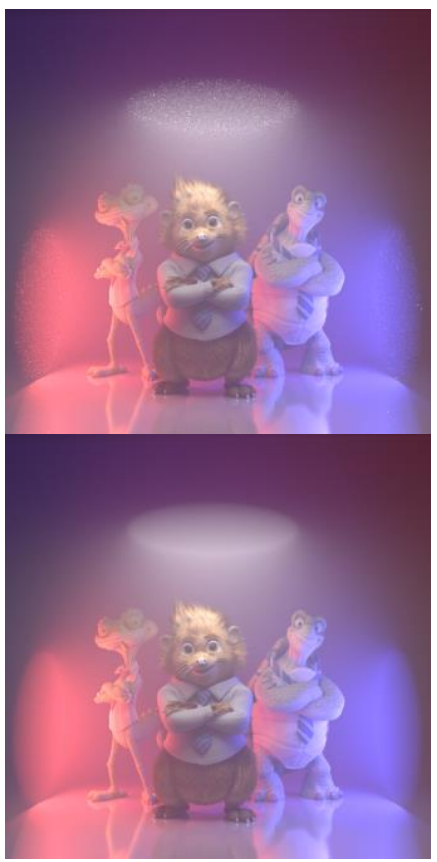

Figure 2. Colored disk lights (invisible) rendered in Arnold using area sampling (left) and our tabulated low distortion map (right) with 256samples/pixel.

Figure 1. A unit square sampling pattern distributed over a disk solid angle projection using (from left to right) surface-area sampling, rejection sampling, our parallel, radial and low-distortion mapping. The points are colored according to their UV coordinates on the unit square to illustrate the continuity of the maps. Our three methods result in uniform distribution of samples on the projected solid angle.

Revista "Jornada de Jóvenes Investigadores del I3A", vol. 6 (Actas de la VII Jornada de Jóvenes Investigadores del I3A - 8 de junio de 2018). ISSN 2341-4790 